\title{
Calcul numérique des tunnels boulonnés par une méthode d'homogénéisation
}

D. BERNAUD

G3S-LMS,

École Polytechnique,

Palaiseau

\section{P. DE BUHAN}

S. MAGHOUS

Laboratoire de Mécanique des Solides

IURA 317 CNRS),

ENPC-CERCSO,

Noisy-Le-Grand
Adoptant le point de vue macroscopique, on décrit dans cet article une simulation numérique de la convergence d'un tunnel renforcé par boulonnage radial et/ou en front de taille. Les relations de comportement anisotrope du matériau homogénéisé, préalablement écrites, sont introduites dans un code numérique par éléments finis adapté pour la modélisation des phases d'excavation. En particulier, on y implante les formules de projection sur le domaine d'élasticité, nécessaires pour la résolution de l'algorithme itératif en plasticité.

Une étude de cas est ensuite entreprise pour simuler les étapes d'excavation/renforcement d'un tunnel et calculer sa convergence au fur et à mesure que le front avance. En sus des deux modes de boulonnage, la prise en compte d'un soutènement en paroi est examinée, mettant en évidence l'interaction entre les différentes composantes de l'ouvrage : massif-boulons-soutènement. Des indications importantes sur les paramètres pertinents du renforcement ont pu être dégagées de cette étude, fournissant des éléments pour le choix d'un schéma de renforcement optimal.

De par sa souplesse et son efficacité, cette méthode constitue un outil de calcul adapté au dimensionnement d'ouvrages géotechniques renforcés par des inclusions régulièrement réparties.

\section{Numerical design of bolt reinforced tunnels by a homogenization method}

This paper is devoted to the numerical simulation of the convergence of a tunnel reinforced by both radial and horizontal bolts. Adopting an anisotropic elastic-plastic constitutive law for the bolted rockmass regarded as a homogenized material, the numerical implementation of such a model in a finite element. computer code specifically devised for calculating the advancement of tunnels, is described.

A parametric study is undertaken by means of this numerical tool, varying some relevant parameters such as the bolt density, the length of bolts or the delay of placement of radial bolts behind the facing. The combination of such a reinforcement technique with a classical lining support system is also investigated by this numerical method, thus providing some general guidelines for the optimal design of reinforcement pattern in order to significantly reduce the convergence at both the tunnel wall and tunnel facing.

It appears that such a numerical approach, developed here in the case of reinforced tunnels, is liable to become a quite suitable tool for dealing with various kinds of geotechnical structures where regularly distributed inclusions are involved. 


\section{Introduction}

La stabilisation des ouvrages souterrains, tels que les tunnels creusés dans des massifs rocheux, demeure une préoccupation majeure de l'ingénieur géotechnicien. En effet, en plus de l'exigence d'un gabarit imposé et d'une durée de vie élevée, celui-ci doit concilier les aspects liés à la technique et la sécurité à ceux concernant l'économie du projet. Le choix et le dimensionnement d'un mode de soutènement adéquat s'avère une étape essentielle du projet.

Dans ce dernier registre, et suite au développement des techniques de renforcement en mécanique des sols, l'utilisation d'inclusions métalliques (boulons) comme système de renforcement dans le domaine des tunnels est devenue de plus en plus courante. Si techniquement et technologiquement ce système est assez bien maîtrisé, sa modélisation du point de vue mécanique et, plus particulièrement, la modélisation de l'interaction massif/inclusion demeure mal connue.

La nature fortement hétérogène du milieu renforcé par boulonnage constitue la source principale des difficultés rencontrées lors d'une modélisation numérique par éléments finis directe. En effet, la taille des élé- ments (mailles) dans la zone concernée par le boulonnage devant être beaucoup plus petite que la longueur caractéristique du renforcement (distance entre deux boulons successifs), ceci conduit à des problèmes de dimension déraisonnable, même pour de puissants calculateurs.

L'objectif de cette contribution est de présenter une alternative pour s'affranchir de cette difficulté, ayant pour cadre celui de la méthode d'homogénéisation des milieux périodiques. En effet, lorsque la densité du boulonnage est suffisamment grande, cette approche heuristique permet de substituer au milieu boulonné hétérogène initial, un milieu homogène équivalent à l'échelle macroscopique. Ainsi, les boulons n'ont plus donc à être considérés séparément.

Cette approche, déjà mise en œuvre et éprouvée dans le cas des sols renforcés (voir par exemple de Buhan et al. (1989) ou de Buhan et Salençon (1990)), s'est révélée très satisfaisante pour décrire à l'échelle macroscopique les capacités de résistance de telles structures. Son principe général a été appliqué pour la première fois pour l'étude d'une cavité renforcée par boulons, en conditions conjuguées d'axisymétrie et de déformation plane, par Wullschläger et Natau (1983, 1987). Plus récemment, Greuell (1993) et Greuell et al. (1994) ont proposé une solution analytique pour le

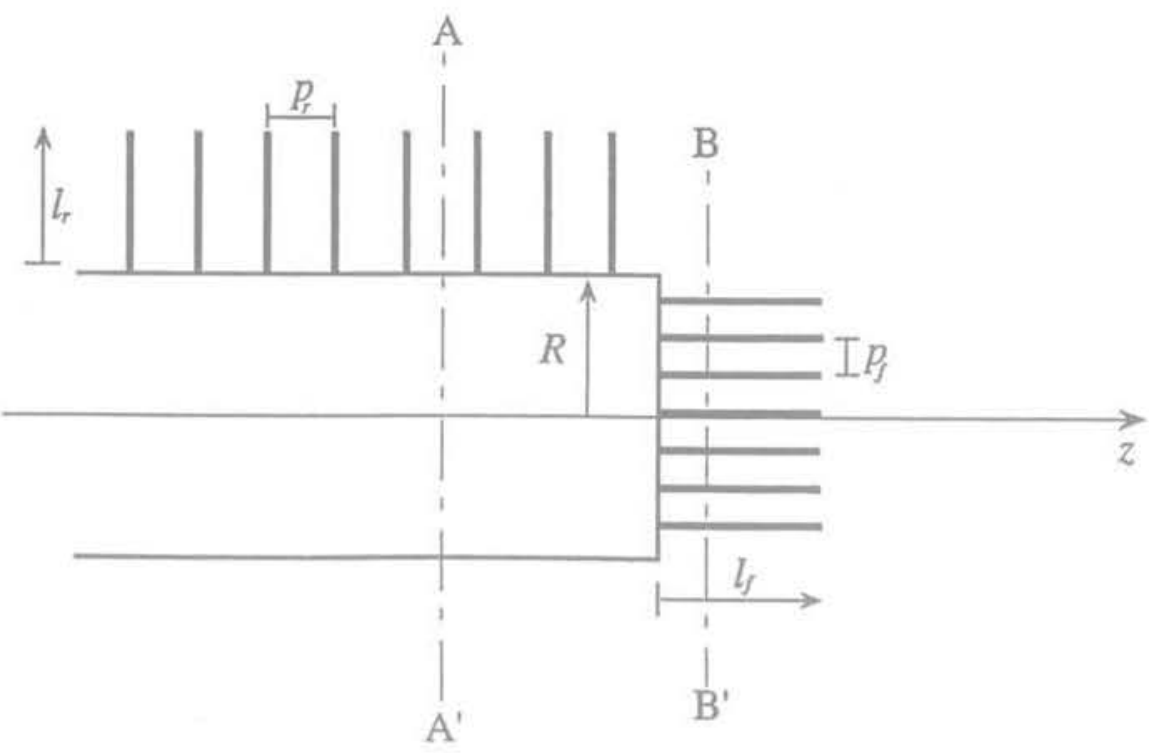

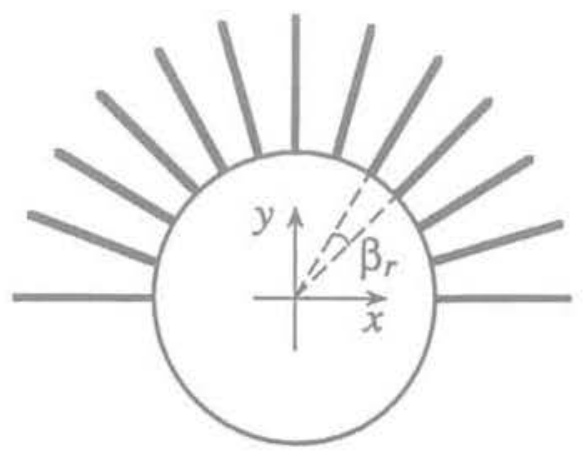

section $\mathrm{AA}^{\prime}$

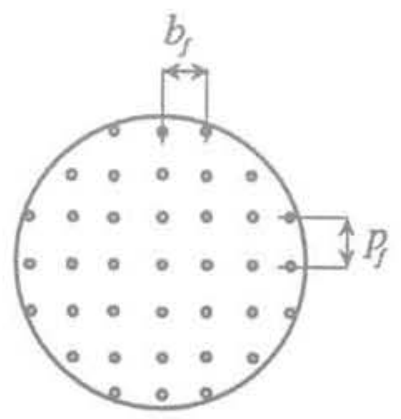

section $\mathrm{BB}^{\prime}$

Fig.1 Caractéristiques géométriques du problème. Geometrical characteristics of the problem. 
même problème avec une loi élastoplastique anisotrope pour modéliser le comportement du massif boulonné en tant que milieu homogénéisé.

Nous proposons une extension de ce dernier travail, permettant de tenir compte de géométries et conditions de chargements plus complexes. Cette extension repose sur la modélisation du tunnel renforcé et la mise en œuvre numérique par éléments finis, une loi de comportement pour ce milieu homogénéisé ayant été auparavant formulée.

\section{2}

\section{Le tunnel boulonné comme un milieu homogénéisé}

Le tunnel considéré, de section circulaire et rayon $\mathrm{R}$, est excavé dans un milieu homogène et isotrope, Il est renforcé par des boulons, disposés au fur et à mesure que le front de taille avance, et supposés adhérer parfaitement au massif. Le boulonnage est soit radial, soit frontal (boulons disposés en front de taille). Ces deux modes de boulonnage peuvent exister individuellement ou conjointement (association des deux modes). Les caractéristiques géométriques du problème sont définies sur la figure 1:

- Boulons radiaux : ils sont placés perpendiculairement à l'axe $\mathrm{zz}^{\prime}$ du tunnel avec un espacement horizontal p (espacement suivant l'axe précédent) et un espacement angulaire $\beta$ (dans la section transversale). Leur longueur, mesurée parallèlement à la direction radiale

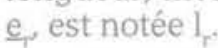

- Boulons frontaux : disposés au front parallèlement à l'axe du tunnel $\mathrm{zz}^{\prime}$, leur longueur est notée $\mathrm{I}$. Ils sont régulièrement répartis dans la section constituant le front, de telle sorte que leurs points d'émergence dans cette section forment par exemple un réseau de mailles rectangulaires $p_{f} \times b_{r}$

Afin d'alléger le texte, on notera dans toute la suite par (ZR) la zone du massif concernée par le boulonnage radial, et par (ZF) celle concernée par le boulonnage frontal.

Supposons maintenant que le renforcement, aussi bien radial que frontal, soit suffisamment dense, c'està-dire que les quantités $\beta / \pi, p / R, p / R$ et $b / R$ soient très petites devant l'unité. Alors, le massif renforcé peut être considéré à l'échelle macroscopique (échelle de l'ouvrage) comme un milieu localement orthotrope autour de l'axe radial e pour (ZR), et homogène orthotrope autour de l'axe du tunnel e pour (ZF). Cette procédure d'homogénéisation simplifie considérablement le problème initial, puisque les constituants du massif renforcé n'ont plus à être considérés séparément, ce qui évite les difficultés de discrétisations très fines rencontrées lors d'une approche par éléments finis directe, rendues nécessaires pour tenir compte de la forte hétérogénéité de la zone renforcée.

Les volumes élémentaires représentatifs, prismatique pour (ZR) et parallélépipédique pour (ZF) (Fig. 2), permettent de définir les paramètres pertinents du renforcement :

- La proportion volumique du renforcement $\eta$, définie comme le rapport entre l'aire de la section transversale du boulons $\mathrm{S}_{\mathrm{r}}$ ou $\mathrm{S}_{\mathrm{r}}$ et celle de la section correspondante du volume representatif :

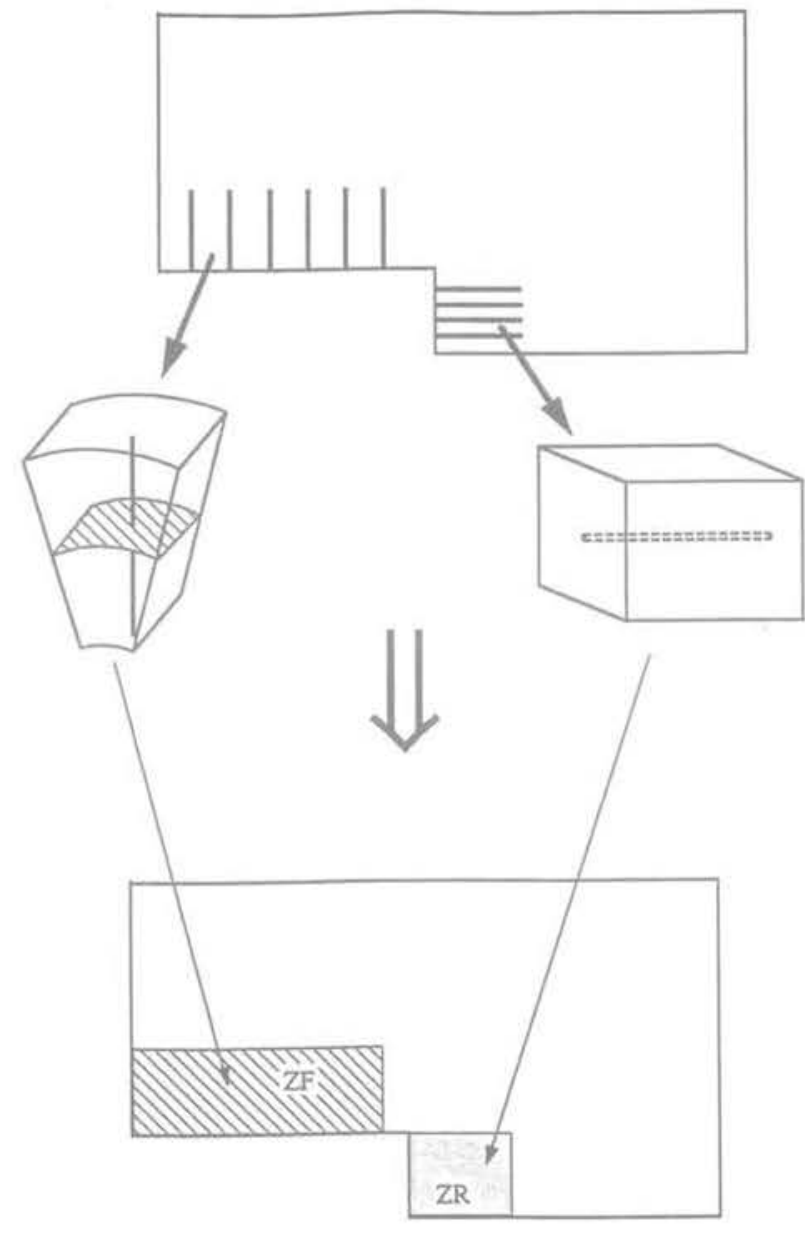

FIG.2 Volumes représentatifs et problème homogénéisé.

Representative elementary volumes and homogenized problem.

$$
\eta= \begin{cases}\eta_{r}(r)=\frac{S_{r}}{p_{r} b_{r} r} & \text { pour (ZR) } \\ \eta_{f}=\frac{S_{f}}{p_{f} b_{f}} & \text { pour (ZF) }\end{cases}
$$

Ce paramètre adimensionnel est constant pour (ZF), alors qu'il décroit en fonction de la distance r à l'axe du tunnel pour (ZR) (écartement entre les boulons radiaux croissant au fur et à mesure que l'on s'éloigne de la paroi du tunnel).

- La densité de renforcement $\delta$, définie par le nombre de boulons par unité de surface de la paroi du tunnel $(r=R)$ pour ( $Z R$ ), et par unité de surface du front de taille pour $(\mathrm{ZF})$ :

$$
\delta= \begin{cases}\delta_{r}=\frac{1}{p_{r} \beta_{r} R} & \text { pour (ZR) } \\ \delta_{f}=\frac{1}{p_{f} b_{f}} & \text { pour (ZF) }\end{cases}
$$

$\delta$ s'exprime bien entendu en boulons $/ \mathrm{m}^{2}$. 
La formulation de la loi de comportement du matériau homogénéisé à partir de celle de chacune des composantes du composite initial est conduite selon la procédure illustrée par la figure suivante:

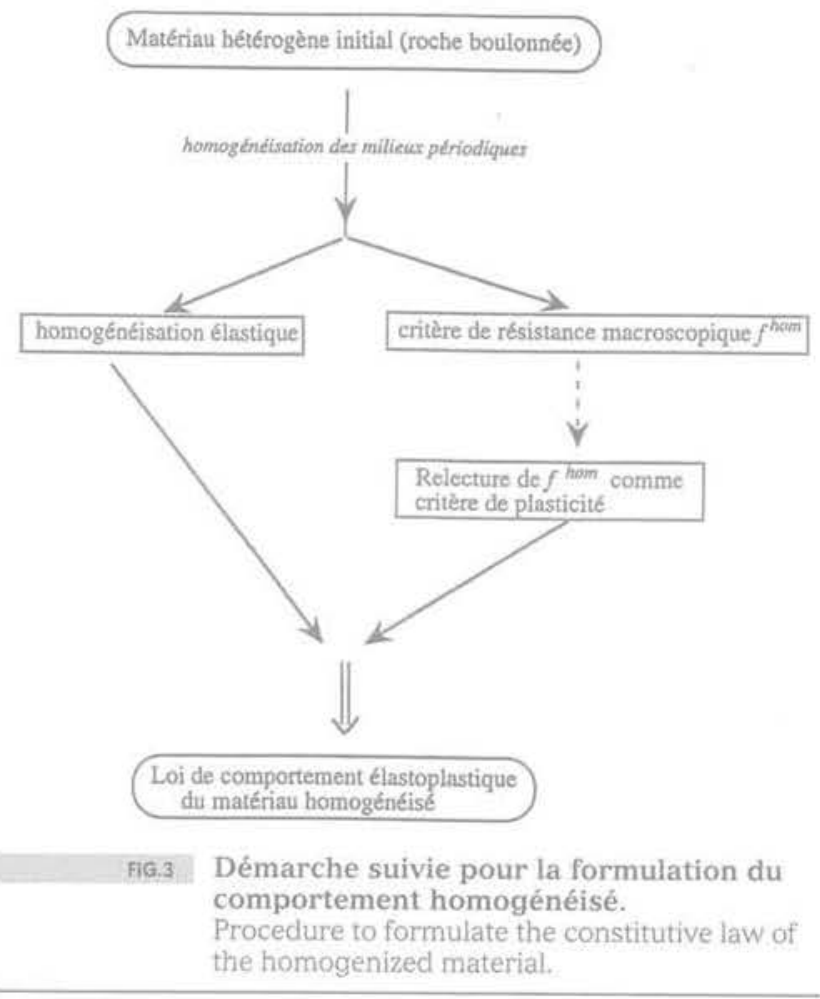

\section{Élasticité du milieu homogénéisé}

La détermination du tenseur d'élasticité de ce milieu constitue la première étape vers la construction d'une loi de comportement du massif boulonné.

Les constituants de celui-ci sont supposés à propriétés élastiques linéaires et isotropes, caractérisées par :

$-E_{m}$ et $v_{m}$ (module d'Young et coefficient de Poisson) pour le massif. On notera $A_{m}$ le tenseur d'élasticité correspondant:

$-E_{r} v_{r}$ et $E_{p}, v_{f}$ les modules d'Young et coefficients de Poisson respectifs du matériau de renforcement constituant les boulons dans (ZR) et dans (ZF).

Dans le cas où simultanément, la proposition volumique de renforcement est très faible $(\eta<<1)$ et la rigidité des boulons est beaucoup plus grande que celle du massif $\left(E_{r} \gg E_{m}\right.$ et $\left.E_{f} \gg E_{m}\right)$, on peut montrer (Greuell (1993)) que le matériau renforcé se comporte à l'échelle macroscopique, comme un milieu élastique isotrope transverse autour de l'axe $\underline{e}_{t}$ pour (ZR) et autour de $\underline{e}_{t}$ pour (ZF). La relation entre les tenseurs \& macroscopiques $)$ de contrainte $\underline{\underline{\Sigma}}$ et de déformation $\underline{\underline{\underline{E}}}$ s'exprimant par :

$$
\underline{\underline{\Sigma}}=\underline{\underline{\underline{\underline{\underline{A_{\mathrm{m}}}}}}}: \underline{\underline{\underline{E}}}+K \in \in_{\mathrm{il}} \underline{\varepsilon}_{i} \otimes \underline{\varepsilon}_{i}
$$

où l'indice $i$ et le coefficient $K$ désignent:

$. i=r$ et $K=\eta_{r}(r) E_{r}$ pour tout point de (ZR).

$\mathrm{i}=\mathrm{z}$ et $\mathrm{K}=\eta_{\mathrm{f}} \mathrm{E}_{\boldsymbol{r}}$ pour tout point de $(\mathrm{ZF})$.

Directement proportionnel à la proportion volumique de renforcement $\eta$, le paramètre de renforcement $\mathrm{K}$ est donc constant dans (ZF) et décroît comme $1 / r$ dans (ZR).

Le second membre de la relation (3) est la somme de deux termes : le premier correspond à l'élasticité du massif non renforcé, tandis que le second traduit l'effet renforçant des boulons, qui n'interviennent donc que selon la direction i. Cette relation peut être réécrite en introduisant le tenseur d'élasticité du matériau homogénéisé de la manière suivante :

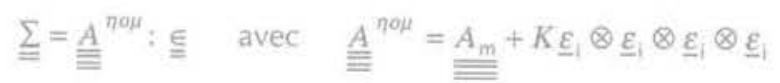

Ce résultat est en accord avec ceux obtenus par Sawicki (1978) ou par Gerrard (1982-a, 1982-b). Le précédent tenseur peut être caractérisé par les paramètres suivants :

- Le module d'Young longitudinal (selon la direction de renforcement $\underline{e}_{\text {i }}$ )

$$
E_{1}^{\text {hom }}=E_{\text {m }}+K
$$

- Le module d'Young transversal (à la direction de renforcement)

$$
E_{t}^{\text {hom }}=E_{m} \frac{E_{m}+K}{E_{m}+\left(1-v_{m}^{2}\right) K}
$$

- Les modules de cisaillement

$$
\mu_{l}^{\text {hom }}=\mu_{t}^{\text {hom }}=\mu_{m}
$$

\section{4}

\section{Loi élastoplastique du massif boulonné}

Comme indiqué sur le schéma de principe de la figure 3 , la construction d'une telle loi se fait en deux étapes : détermination du critère de résistance de ce composite à partir de celui de ses composants (roche, boulons), adoption de ce critère comme critère de plasticité parfaite avec loi d'écoulement associée.

\section{1}

\section{Critère de résistance macroscopique}

La détermination de ce critère ressortit à la théorie de l'homogénéisation en calcul à la rupture, dont l'application aux matériaux composites à structure périodique peut être trouvée dans de Buhan (1986), de 
Buhan et Salençon (1990) ou de Buhan et Taliercio (1991). En étendant les résultats obtenus par ces derniers auteurs, le critère de résistance $f^{\text {hom }}$ de la zone boulonnée peut être exprimé par :

$$
f^{\text {hom }}(\underline{\underline{\Sigma}}) \leq 0 \Leftrightarrow\left\{\begin{array}{l}
\underline{\underline{\underline{\Sigma}}}=\underline{\underline{\sigma}}_{m}+\sigma \underline{\mathrm{e}}_{i} \otimes \underline{\mathrm{e}}_{i} \\
\text { avec } \\
f_{m}\left(\underline{\underline{\sigma}}_{m}\right) \leq 0 \text { et } \sigma \in I
\end{array}\right.
$$

où $f_{\mathrm{f}}$ désigne le critère de résistance de la roche non renforcée, et : (ZF).

I'indice i vaut $r$ et $z$ respectivement dans (ZR) et

I est l'intervalle:

$$
\mathrm{I}=\left[-\mathrm{k} \eta \sigma_{0}, \eta \sigma_{0}\right]
$$

$\sigma_{0}$ est la limite en traction simple du matériau constitutif des boulons, et $\mathrm{k}$ un nombre adimensionnel compris entre 0 et 1 , réduisant la résistance en compression pour tenir compte du phénomène de flambement. Bien entendu, $\sigma_{0}$ et k peuvent être éventuellement différents selon que l'on considère des boulons utilisés pour le renforcement radial ou frontal. Noter que la quantité $\eta \sigma_{0}$ apparaît comme la résistance en traction des boulons par unité de surface transversale au point considéré.

Le domaine $G^{\text {hom }}$ correspondant au critère f fom s'interprète géométriquement, dans l'espace des contraintes macroscopiques, comme l'enveloppe convexe des deux domaines obtenus par translation du domaine $G_{m}$ (correspondant au critère $f_{m}$ ) parallèlement à l'axe $\Sigma$, des quantités algébriques - $k \eta \sigma_{0}$ et $\eta \sigma_{0}$ (Fig. 4). L'effet de renforcement du massif par les boulons se traduisant par ces deux translations.

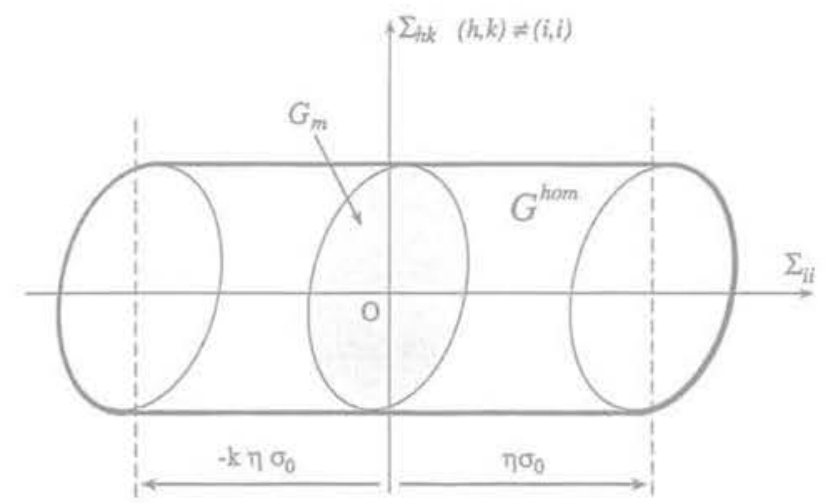

คG.4 Interprétation géométrique du domaine Grom.

Geometrical interpretation of the macroscopic strength domain.

\section{2}

\section{Loi de comportement élastoplastique de la roche boulonnée}

Le principe de la construction d'une telle loi découle du raisonnement heuristique qui consiste à faire une relecture du critère de résistance macroscopique $f^{\text {hom }}$ en l'adoptant comme critère de plasticité pour le matériau homogénéisé. Le comportement de ce dernier est alors modélisé par une loi élastique parfaitement plastique associée, le domaine $\mathrm{G}^{\text {hom }}$ constituant alors le domaine d'élasticité.

Cette loi se traduit dans le cadre des petites perturbations par :

$$
\underline{\underline{E}}=\underline{\underline{E}}^{e}+\underline{\underline{E}}^{p}
$$

où 自 est le taux des déformations totales, $\underline{\underline{\underline{e}}}^{e}$ la part élastique qui est reliée à $\Sigma$ par:

$$
\underline{\underline{\Sigma}}=\underline{\underline{\underline{\underline{A}}}}^{\text {hom }}: \dot{\underline{\epsilon}}^{\mathrm{e}}
$$

et $\in^{P}$ la partie plastique, qui dérive de la loi de normalité par:

$$
\underline{\underline{\epsilon}}^{p}=\dot{w} \frac{\partial f^{\text {hom }}}{\partial \underline{\underline{\Sigma}}}(\underline{\underline{\Sigma}}), \quad \dot{w} \geq 0
$$

où $\underline{\underline{\underline{w}}} \geq 0$ est le multiplicateur plastique.

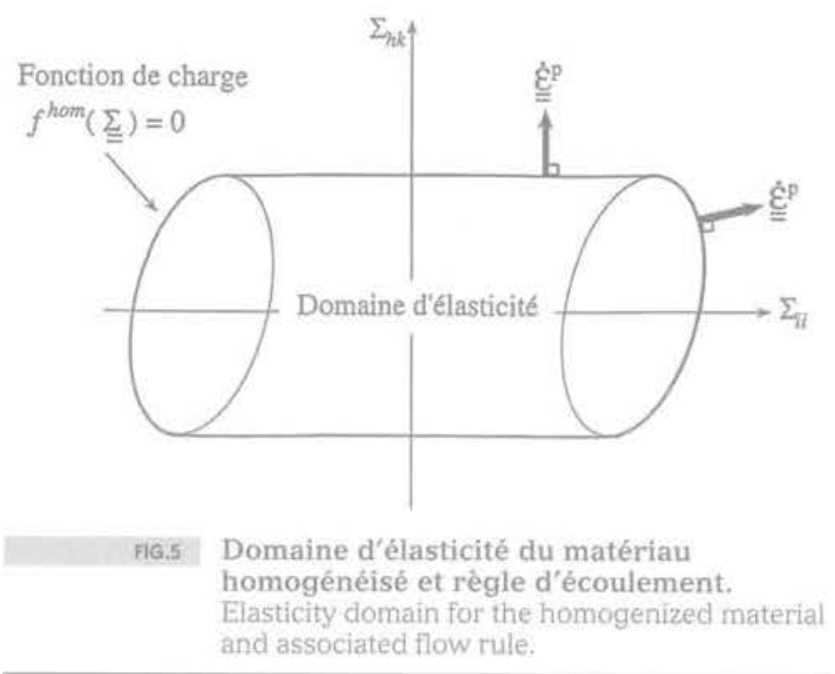

5

\section{Formulation explicite des relations de comportement}

La fonction de charge du matériau homogénéisé définie par (8) peut être réécrite par :

$$
f^{\text {hom }}(\underline{\underline{\Sigma}})=\min _{\sigma \in l} f_{m}\left(\underline{\underline{\Sigma}}-\sigma \underline{e}_{i} \otimes \underline{e}_{i}\right)
$$

Rappelons que l'intervalle I est défini par (9). On montre (voir Bernaud et al., 1995) que :

$$
f^{\text {hom }}(\underline{\underline{\Sigma}})=f_{m}\left(\underline{\underline{\Sigma}}-\sigma^{*} \underline{e}_{i} \otimes \underline{e}_{i}\right)
$$


où

$$
\sigma^{*}= \begin{cases}-k \eta \sigma_{0} & \text { si } x_{0} \leq-k \eta \sigma_{0} \\ x_{0} & \text { si } x_{0} \in I \\ \eta \sigma_{0} & \text { si } x_{0} \geq \eta \sigma_{0}\end{cases}
$$

$\mathrm{x}_{0}$ étant I'unique solution (convexité de $f_{\mathrm{m}}$ ) sur $\mathbb{R}$ de l'équation en $\mathrm{x}$ :

$$
\frac{\partial f_{m}}{\partial \sigma_{i j}}\left(\underline{\underline{\Sigma}}-x \underline{e}_{i} \otimes \underline{e}_{i}\right)=0
$$

$\sigma^{*}$ est donc la projection de $x_{0}$ sur l'intervalle I. Noter que $\sigma^{*}=\sigma^{*}(\underline{\underline{\Sigma}})$, puisque $x_{0}=x_{0}(\underline{\underline{\Sigma}})$.

Afin de compléter la caractérisation de la loi de comportement, et plus particulièrement la formulation de la loi d'écoulement plastique (12), il convient

d'expliciter l'expression de $\frac{\partial f^{\text {hom }}}{\partial \underline{\underline{\underline{\Sigma}}}}(\underline{\underline{\Sigma}})$.

Celle-ci a été établie dans Bernaud et al. (1995) :

$$
\frac{\partial f^{\text {hom }}}{\partial \underline{\underline{\underline{\Sigma}}}}(\underline{\underline{\underline{\Sigma}}})=\frac{\partial f_{m}}{\partial \underline{\underline{\sigma}}}\left(\underline{\underline{\Sigma}}-\sigma^{*} \underline{\underline{e}}_{i} \otimes \underline{\underline{e}}_{i}\right)
$$

Ces relations vont maintenant être illustrées dans le cas où le matériau constitutif de la roche non boulonnée obéit à un critère de Drücker-Prager. C'est-à-dire que $f_{\mathrm{m}}$ s'écrit :

$$
f_{m}(\underline{\underline{\sigma}})=\sqrt{\frac{3}{2}}\|\triangleq\|+\alpha_{m}\left(\operatorname{tr} \underline{\underline{\sigma}}-\sigma_{m}\right)-\sigma_{m} \leq 0
$$

où $\underline{\underline{S}}=\operatorname{dev}(\underline{\underline{\sigma}})$ est la partie déviatorique de $\underline{\underline{\sigma}} \cdot \sigma_{m}$ désigne la limite élastique du matériau en traction simple. $\alpha_{\mathrm{m}}$ est un paramètre compris entre 0 (critère de von Mises) et 1, qui traduit la dépendance du critère vis-à-vis de la contrainte hydrostatique. $\|\underline{\underline{s}}\|=(\underline{\underline{\underline{s}}}: \underline{\underline{\underline{s}}})^{4 / 2}$.

En notant $\underline{\underline{S}}=\operatorname{dev}(\underline{\underline{\Sigma}})$, la résolution de l'équation (16) conduit à :

$$
x_{0}=\frac{3}{2}\left(S_{i i}+\chi\right)
$$

avec

$$
\begin{aligned}
& \chi=\sqrt{\frac{3}{2}} \frac{\alpha_{m}}{\sqrt{1-\alpha_{m}^{2}}} \| \underline{S}-\frac{1}{2} S_{i j}\left(3 \underline{e}_{i} \otimes \underline{e}_{i}-\right. \\
& \left.\underline{e}_{r} \otimes \underline{e}_{r}-\underline{e}_{\theta} \otimes \underline{e}_{\theta}-\underline{e}_{2} \otimes \underline{e}_{2}\right) \|
\end{aligned}
$$

La valeur de $\sigma^{*}$ est déduite de celle de $x_{0}$ par les relations (15), si bien que l'expression de $f^{\text {hiom }}(\underline{\underline{\Sigma}})$ est complètement déterminée par (14),

D'autre part, et en vertu de (17) :

$$
\frac{\partial f^{h o m}}{\partial \underline{\underline{\underline{\Sigma}}}}(\underline{\underline{\underline{\Sigma}}})=\alpha_{\mathrm{m}} \underline{\underline{1}}+\sqrt{\frac{3}{2}} \frac{\underline{\underline{\underline{S}}}-\underline{\underline{\underline{S}}}^{*}}{\left\|\underline{\underline{S}}-\underline{\underline{\underline{S}}}^{*}\right\|}
$$

\section{Mise en cuvre numérique}

Les relations de comportement présentées ci-dessus ont été introduites dans le code de calcul par éléments finis GEOMEC91 (Bernaud, 1991). Les formules de projection sur le convexe d'élasticité ont été établies dans Bernaud et al. (1995) à partir de la formule (21) et incorporées dans ce code. De telles formules sont en effet requises pour l'utilisation de l'algorithme itératif de plasticité (Nguyen Qoc Son, 1977).

Remarquons que le calcul des matrices de rigidité élémentaires correspondant à (ZR) nécessite un traitement spécifique. En effet, outre le caractère anisotrope des paramètres d'élasticité, ceux-ci dépendent du point considéré (variations en 1/r).

\section{6}

\section{Simulation numérique du tunnel boulonné}

\section{1}

\section{Position du problème}

Le tunnel considéré, de section circulaire (rayon R), est supposé creusé à grande profondeur $\mathrm{H} \gg \mathrm{R}$ dans une roche homogène et isotrope. Avant l'excavation du tunnel, le champ de contrainte géostatique régnant dans le massif à cette profondeur est supposé isotrope et homogène $\sigma_{0}=-P_{0} 1$. En effet, l'effet de la pesanteur peut être négligé dans une zone proche du tunnel $\left(\gamma \mathrm{R}<<\mathrm{P}_{\alpha}\right.$ où $\gamma$ désigne le poids volumique du massif $)$ (Fig. 6)

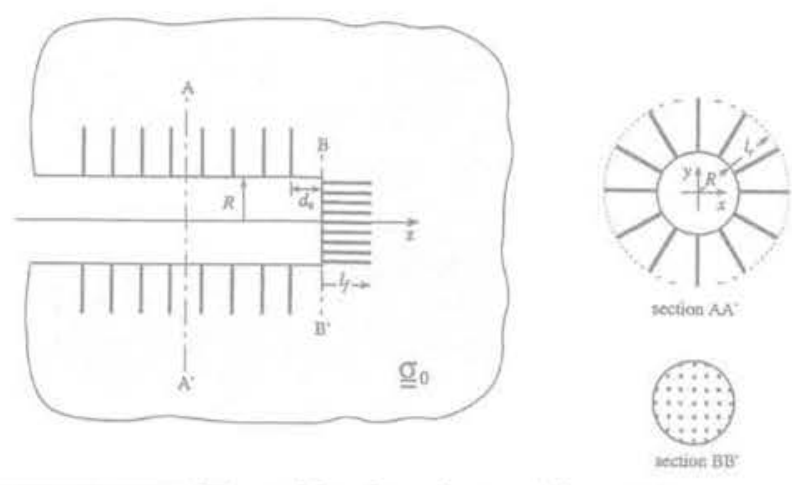

FG.6 Tunnel boulonné creusé à grande profondeur dans un milieu isotrope. Bolt supported tunnel excavated at great depth in an isotropic rockmass.

Les deux schémas de boulonnage, considérés séparément ou en association, sont étudiés : boulons radiaux (caractéristiques $1_{r}, \delta, S_{r}$ ) et boulons frontaux (caractéristiques $\mathrm{I}_{f} \delta_{r}, \mathrm{~S}$ ). Les boulons radiaux sont disposés autour de la paroi du tunnel suivant toutes les directions radiales (section $\mathrm{AA}^{\prime}$ ) à partir d'une distance $\mathrm{d}_{0}$ du front de taille. Les boulons frontaux sont placés tout autour du centre de symétrie $\mathrm{O}(\mathrm{r}=0)$ du disque symbolisant le front de taille. Leurs intersections avec ce disque forment un nuage de points régulièrement répartis (section $\left.\mathrm{BB}^{\prime}\right)$. 
Suivant la procédure d'homogénéisation décrite en section 2, et sous les hypothèses d'ordre géométrique mentionnées ci-dessus, les zones renforcées sont donc modélisées par:

- un anneau cylindrique d'axe $\mathrm{Oz}$, de rayon intérieur R et extérieur $\mathrm{R}+\mathrm{I}_{\mathrm{r}}$;

- un cylindre, d'axe $\mathrm{Oz}$, s'appuyant sur le front de taille, de longueur $l_{\text {f }}$ et de rayon $\mathrm{R}$,

constitués de matériaux homogénéisés, localement orthotropes autour des directions $\underline{\mathrm{e}}_{\mathrm{r}}$ et $\underline{\mathrm{e}}_{2}$ respectivement.

Il s'ensuit que le problème tridimensionnel du creusement de ce tunnel, peut être traité comme un problème axisymétrique dans les axes ( $\mathrm{r}, \mathrm{z}$ ).

L'objectif de cette étude est de déterminer les champs de contrainte et de déplacement induits par le processus d'excavation, et plus particulièrement de quantifier le rôle de renforcement par boulonnage dans la réduction de la convergence du tunnel.

La simulation numérique des étapes de creusement/avancement du tunnel est modélisée par la méthode "d'activation/désactivation » (Hanafy and Emery, 1980) implantée dans le code GEOMEC91. On pourra se référer à Bernaud et al. (1995) pour les détails concernant l'adaptation de cette technique et sa mise en cuvre numérique dans le cas du renforcement par boulonnage. Bien entendu, cette technique permet de prendre en compte l'association boulonnage/soutènement en paroi du tunnel, tel qu'une couche de béton projeté qui accompagne usuellement le boulonnage radial.

Remarque: Tous les calculs qui seront présentés par la suite ont été effectués en considérant la longueur 1 . des boulons frontaux constante tout au long du processus d'excavation. En réalité, ceux-ci étant constitués d'un matériau fibre de verre, sont " cassés 》 (sur une longueur égale au pas de creusement) et ( excavés 》 avec la roche au fur et à mesure que le tunnel avance. En toute rigueur, à un instant donné, ces boulons n'ont donc pas tous la même longueur.

\section{2}

\section{Validation du code numérique}

En vue de valider l'outil numérique, une série de calculs a été effectuée et les résultats comparés à ceux donnés par Greuell (1993). Ils correspondent au cas d'un tunnel étudié en déformation plane avec conditions de symétrie de révolution (i.e. le déplacement est de la forme $\underline{u}(r, \theta, z)=u(r) \underline{e})$, ce qui correspond à un problème $(1 \mathrm{D})$. Le matériau constitutif de la roche non renforcée étant supposé obéir à un critère de Tresca (milieu purement cohérent), et seul le boulonnage radial est considéré.

Il ressort de cette comparaison (voir Bernaud et al., 1995) une parfaite concordance entre les résultats numériques et analytiques aussi bien en ce qui concerne :

- les courbes $\left(\mathrm{P}_{p}, \mathrm{U}_{p}\right)$, où $\mathrm{P}$ désigne la pression appliquée en paroi du tunnel, et $U_{\text {i }}$ sa convergence loin du front $(=-u(r=R) / R)$,

- qu'en ce qui concerne l'influence d'un délai de pose des boulons (radiaux) sur l'équilibre final du tunnel. Celle-ci étant prise en compte via le taux de déconfinement $\lambda_{0}$ (voir Panet et Guellec, 1974).
Remarque: Toujours dans ce même cadre, des calculs numériques 3D axisymétriques ont été effectués dans le cas théorique $\lambda_{0}=0$ (i.e. tunnel excavé dans un massif déjà boulonné !). Les valeurs de la convergence finale loin du front obtenues diffèrent de moins de $1 \%$ de celles données analytiquement par Greuell (1993).

\section{7}

\section{Calculs numériques 3D axisymétriques}

\section{1}

\section{Données relatives aux calculs}

Nous nous intéressons maintenant à la modélisation en conditions d'axisymétrie des phases de creusement du tunnel et pose du renforcement. Rappelons que les principaux paramètres régissant ce phasage sont:

- la distance de pose des boulons radiaux $\mathrm{d}_{0}$;

- le pas d'excavation. Celui-ci est supposé constant dans la modélisation numérique égal à R/3 (à l'exception du premier creusement, où il est pris égal à R).

Tous les calculs présentés par la suite ont été réalisés en utilisant le maillage par éléments finis de la figure 7 (1 298 quadrilatères à 9 nœuds). Il faut souligner que celui-ci est strictement analogue à ceux utilisés en absence de renforcement. Ce qui justifie encore l'emploi d'une démarche d'homogénéisation pour le massif renforcé par boulonnage.

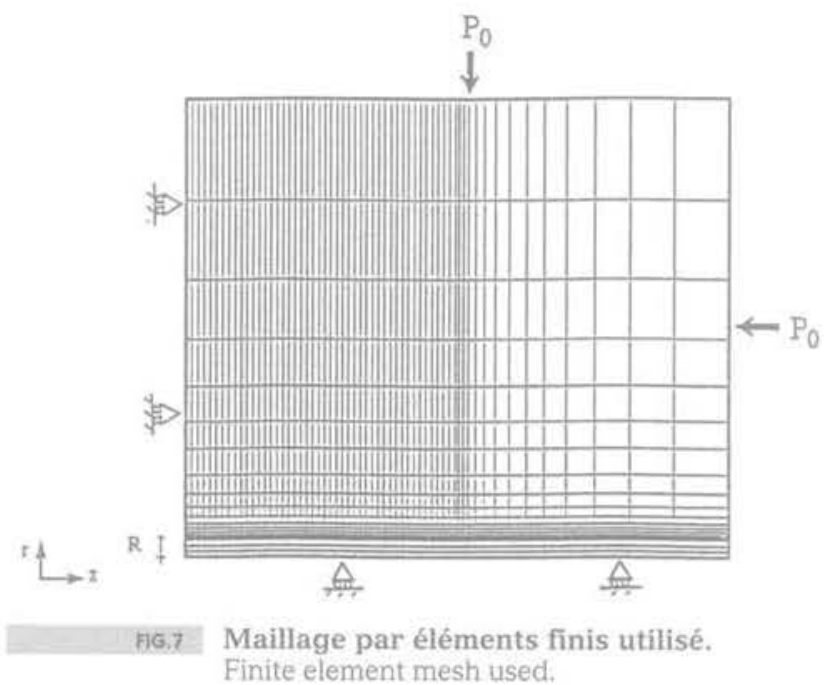

Afin d'apprécier l'influence de certains paramètres sur l'équilibre final de l'ouvrage, une étude paramétrique (non exhaustive) est entreprise dont les résultats sont présentés ci-après.

Les caractéristiques mécaniques considérées pour la roche non renforcée sont fixées à :

$$
\left\{\begin{array}{l}
c_{m}=1,2 \mathrm{MPa}, \varphi_{\mathrm{m}}=4^{\circ} \\
E_{m}=500 \mathrm{MPa}, v_{m}=0,45
\end{array}\right.
$$

(Drücker - Prager) 
Ces caractéristiques correspondent à une roche tendre du type argile de Boom (galerie expérimentale creusée à une profondeur de $200 \mathrm{~m}$ à Mol, Belgique voir à cet effet Bernaud et Rousset,1993) .

La pression initiale de confinement est prise égale à $\mathrm{P}_{0}=4 \mathrm{MPa}$.

Bien que les résultats puissent être exprimés en fonction de paramètres adimensionnels $x / R$ (où $x$ désigne toute grandeur de l'ouvrage homogène à une longueur), le rayon du tunnel est pris égal à $R=3 \mathrm{~m}$. lons :

En ce qui concernent les données relatives aux bou-

\section{- boulons radiaux (acier):}

$$
\left\{\begin{array}{l}
\text { diamètre } D=20 \mathrm{~mm} \\
\text { limite en traction simple } \sigma_{0}=650 \mathrm{MPa} \\
\text { facteur de rèduction en compression } \mathrm{k}=0 \\
E_{\mathrm{r}}=2 \times 10^{5} \mathrm{MPa}
\end{array}\right.
$$

\section{- boulons frontaux (fibre de verre):}

$$
\left\{\begin{array}{l}
\text { diamètre intérieur } D_{i}=10 \mathrm{~mm} \\
\text { diamètre extérieur } D_{c}=40 \mathrm{~mm} \\
\text { limite en traction simple } \sigma_{0}=300 \mathrm{MPa} \\
\text { facteur de réduction en compression } k=0 \\
E_{f}=2 \times 10^{4} \mathrm{MPa}
\end{array}\right.
$$

Remarque : Compte tenu des caractéristiques spécifiques adoptées ici pour la roche et les boulons, les conclusions relatives à l'influence des paramètres de renforcement, qui seront formulées à la suite de l'étude numérique doivent être considérées avec prudence. Elles ne sont pas a priori généralisables à des roches et boulons de caractéristiques mécaniques différentes de celles considérées ici. Toutefois, on peut raisonnablement considérer qu'elles en traduisent bien les tendances.

\section{Influence de la longueur des boulons}

Lorsqu'on ne considère que les boulons radiaux, il a été constaté analytiquement (Greuell, 1993) et numériquement (Bernaud et al., 1995), toutes choses étant égales par ailleurs, qu'au-delà d'une longueur égale au diamètre du tunnel $1>2 R$, l'influence de la longueur des boulons radiaux sur l'ampleur de la convergence du tunnel cessait d'être significative.

Examinons maintenant l'influence de la longeur l. des boulons frontaux, lorsqu'ils constituent le seul mode de renforcement (pas de boulonnage radial). Un premier calcul a été effectué avec une densité $\delta_{f}=1 \mathrm{~b} / \mathrm{m}^{2}$ et des longueurs $1_{\text {s }}$ égale à $\mathrm{R}$ ou $2 \mathrm{R}$. Sur la figure 8 on représente la convergence radiale finale $\mathrm{U}_{\mathrm{f}}=-\mathrm{u}_{\mathrm{f}}(\mathrm{r}=\mathrm{R}, \mathrm{z}) / \mathrm{R}$ en fonction de la distance au front de taille. On constate qu'hormis au voisinage du front de taille, la présence des boulons frontaux n'induit aucune réduction de la convergence en paroi par rapport au cas non renforcé. Loin du front de taille, cette convergence demeure toujours égale à $2,44 \%$.

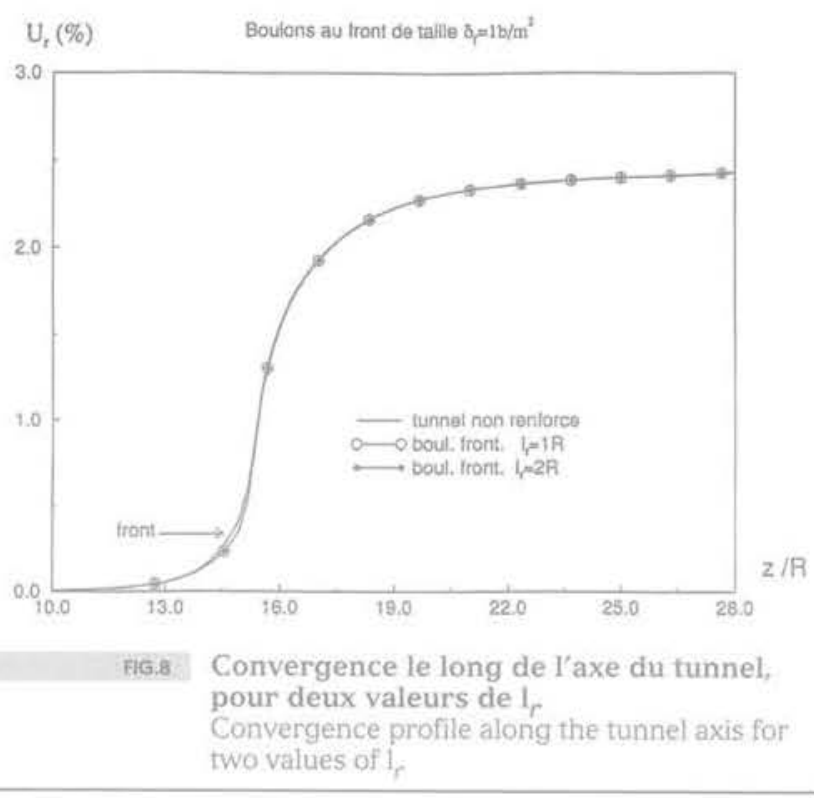

On définit la convergence axiale par le rapport $\mathrm{U}_{2}=-\mathrm{u}_{\mathrm{z}}\left(\mathrm{r}, \mathrm{z}=\mathrm{z}_{\text {front }}\right) / \mathrm{R}$, où $\mathrm{u}_{\mathrm{z}}\left(\mathrm{r}, \mathrm{z}=\mathrm{z}_{\text {frunt }}\right)$ est le déplacement selon e (axe du tunnel) dans le plan du front de taille. Lorsque l'on considère les variations de $\mathrm{U}$, en fonction de $r$, il apparait clairement sur la figure 9 que la présence des boulons frontaux réduit sensiblement cette convergence. Pour fixer les idées, examinons sa valeur maximale (obtenue en $\mathrm{r}=0$, centre de symétrie du front). Elle vaut :

$-1,63 \%$ dans le cas non renforcé;

$-1,31 \%$ en présence des boulons frontaux avec $\delta_{\mathrm{f}}=1 \mathrm{~b} / \mathrm{m}^{2}$ et $\mathrm{l}_{\mathrm{f}}=\mathrm{R}$ ou $2 \mathrm{R}$;

soit une réduction d'environ $20 \%$.

Le premier enseignement déduit de ces résultats est qu'ils semblent indiquer qu'au-delà d'une longueur $\mathrm{L}_{\mathrm{f}}=\mathrm{R}$, la convergence axiale (et bien entendu radiale) n'est plus affectée. Ce résultat est confirmé par des calculs effectués (non présentés ici) en prenant $l_{f}=3 R$ et $5 \mathrm{R}$.

Le second enseignement est qu'il confirme bien le rôle stabilisateur du front de taille d'un renforcement

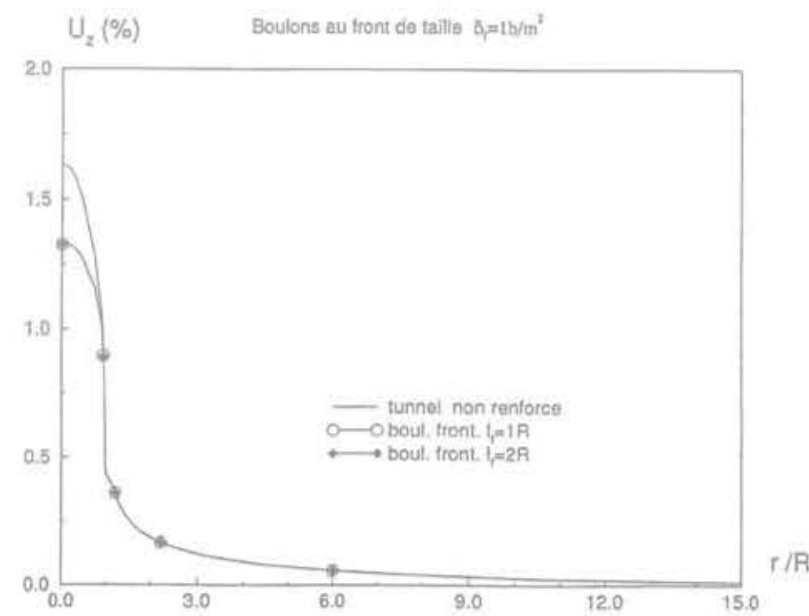

FIG.9 Convergence axiale en fonction de $r$. Axial convergence along the $\mathrm{r}$-axis. 
par boulons frontaux, en "empêchant ») la venue du front (réduction de $\mathrm{U}_{2}$ ), ce qui est fort appréciable en phase de construction.

Le dernier enseignement est qu'utilisé seul, sans l'adjonction d'aucun autre type de renforcement, ce mode de renforcement est inefficace quand il s'agit de réduire la convergence radiale du tunnel loin du front.

$\mathrm{Au}$ terme de cette section, il faut retenir (sous réserve de la remarque faite en fin de la section 7.1) que les longueurs efficaces sont de l'ordre de $\mathrm{R}$ pour les boulons frontaux et de $2 \mathrm{R}$ pour les boulons radiaux. Des calculs effectués en associant les deux modes de boulonnage, pour appréhender un éventuel effet de " couplage », semblent indiquer que ces valeurs ne sont pas modifiées.

\section{3}

\section{Influence de la densité de boulonnage}

Comme précédemment, nous n'examinerons que l'influence de la densité des boulons frontaux, quand ceux-ci sont utilisés seuls. En effet, lorsqu'il s'agit de boulons radiaux seuls, on pourra se référer à Bernaud et al. (1995) où l'influence assez importante de la densité sur la convergence radiale a été étudiée et quantifiée.

Bien entendu, dans le cas examiné de boulonnage frontal seul, cette influence se mesure uniquement sur la convergence axiale. On représente sur la figure 10 cette convergence pour différentes valeurs de la densité $\delta_{p}$, la longueur $\mathrm{l}_{\mathrm{f}}$ étant fixée à $\mathrm{R}$. Comme prévu, ce paramètre affecte significativement la valeur de la convergence axiale. En $r=0$, valant 1,63\% dans le cas non renforcé, elle passe à :

$-1,47 \%$ pour $\delta_{f}=0,5 \mathrm{~b} / \mathrm{m}^{2}$, soit une réduction d'environ $9,5 \%$;

$-1,31 \%$ pour $\delta_{f}=1 \mathrm{~b} / \mathrm{m}^{2}$, soit une réduction d'environ $20 \%$;

$-1,14 \%$ pour $\delta_{f}=2 \mathrm{~b} / \mathrm{m}^{2}$, soit une réduction d'environ $30 \%$.

Combinant les résultats de 7.2 et 7.3 , on constate que dans l'optique du choix d'un schéma de renforcement final, il est plus efficace d'intervenir sur le paramètre densité de boulonnage $\delta$ que sur la longueur L.

$U_{1}(\%) \quad$ Association des deix modes de boulonnege $d_{0}=0$

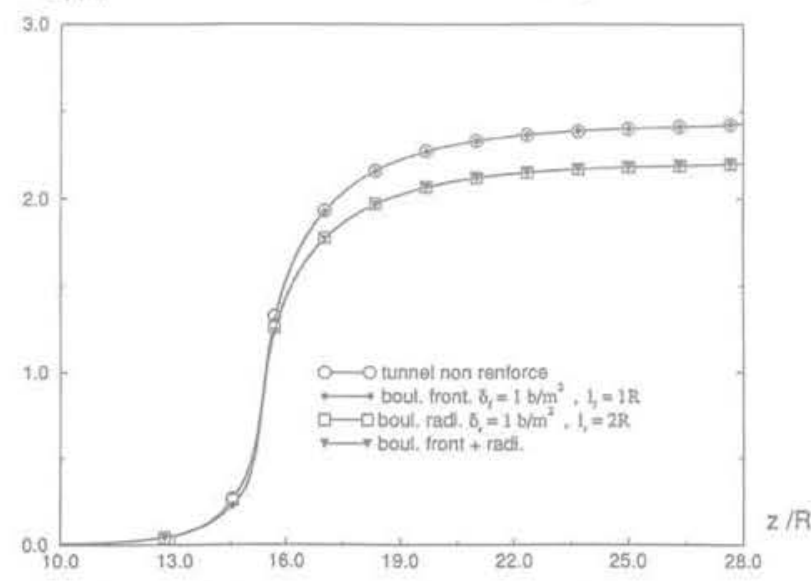

FIG.11-a

Convergence radiale pour l'association des deux modes de boulonnage : $\mathrm{d}_{0}=0$. Radial convergence when associating radial and facing bolts : $\mathrm{d}_{0}=0$.

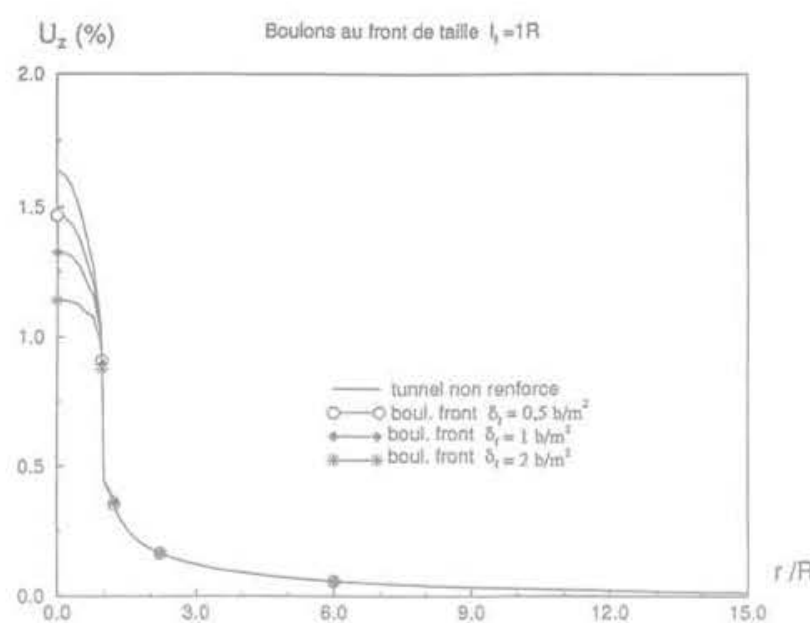

FiG:10 Influence de la densité de boulonnage frontal sur la convergence axiale. Influence of the facing bolt density on the axial convergence.

\section{Association des deux modes de boulonnage}

L'association des boulonnages radial et frontal permet d'intervenir de façon efficace sur l'équilibre final de l'ouvrage, en réduisant simultanément sa convergence radiale et axiale. Nous avons choisi de fixer les paramètres longueur et densité des boulons à :

$$
\begin{aligned}
& -\mathrm{I}_{\mathrm{r}}=2 \mathrm{R} \text { et } \delta_{\mathrm{r}}=1 \mathrm{~b} / \mathrm{m}^{2} \\
& -\mathrm{I}_{\mathrm{f}}=\operatorname{Ret} \delta_{\mathrm{f}}=1 \mathrm{~b} / \mathrm{m}^{2}
\end{aligned}
$$

Le seul paramètre que l'on fasse varier ici est la valeur de $\mathrm{d}_{\alpha}$, distance de pose au front des boulons radiaux. On se restreint aux deux situations $\mathrm{d}_{0}=0$ et $\mathrm{d}_{0}=2 \mathrm{R} / 3$.

\section{- Cas $d_{0}=0$}

Concernant la convergence finale du tunnel et comme signalé auparavant, on constate sur la figure 11-a que les courbes relatives au cas non renforcé et
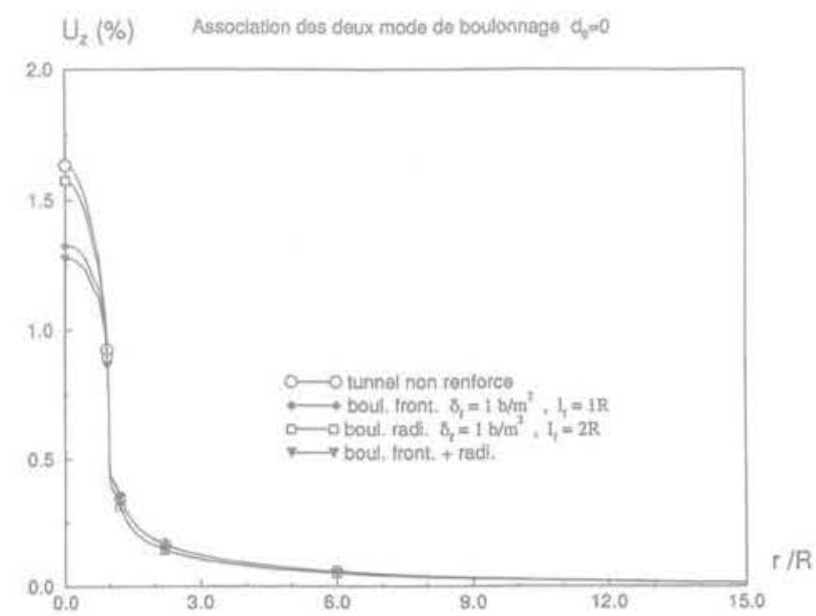

FlG.11-b Convergence axiale pour l'association des deux modes de boulonnage : $\mathrm{d}_{0}=0$. Axial convergence when associating radial and facing bolts : $\mathrm{d}_{0}=0$. 
au cas de boulons frontaux sont confondues (sauf au voisinage du front de taille). Il en est de même pour celles relatives au cas boulons radiaux seuls et au cas boulons radiaux + frontaux.

La valeur de cette convergence loin du front passe de $2,44 \%$ à $2,20 \%$, soit une réduction d'environ $10 \%$. Bien entendu, cette réduction s'amplifie avec la densité $\delta$. A titre d'exemple, cette réduction passe à $20 \%$ lorsque $\delta_{r}=2 \mathrm{~b} / \mathrm{m}^{2}$.

La réduction de la convergence axiale est illustrée sur la figure 11-b, et les valeurs de cette convergence en $\mathrm{r}=0$ sont :

$-1,31 \%$ pour le renforcement frontal seul;

$-1,57 \%$ pour le renforcement radial seul;

$-1,27 \%$ pour l'association des deux modes de renforcement

et comme prévu, l'essentiel de la réduction de cette convergence est dû aux seuls boulons frontaux.

- Cas $d_{0}=2 R / 3$
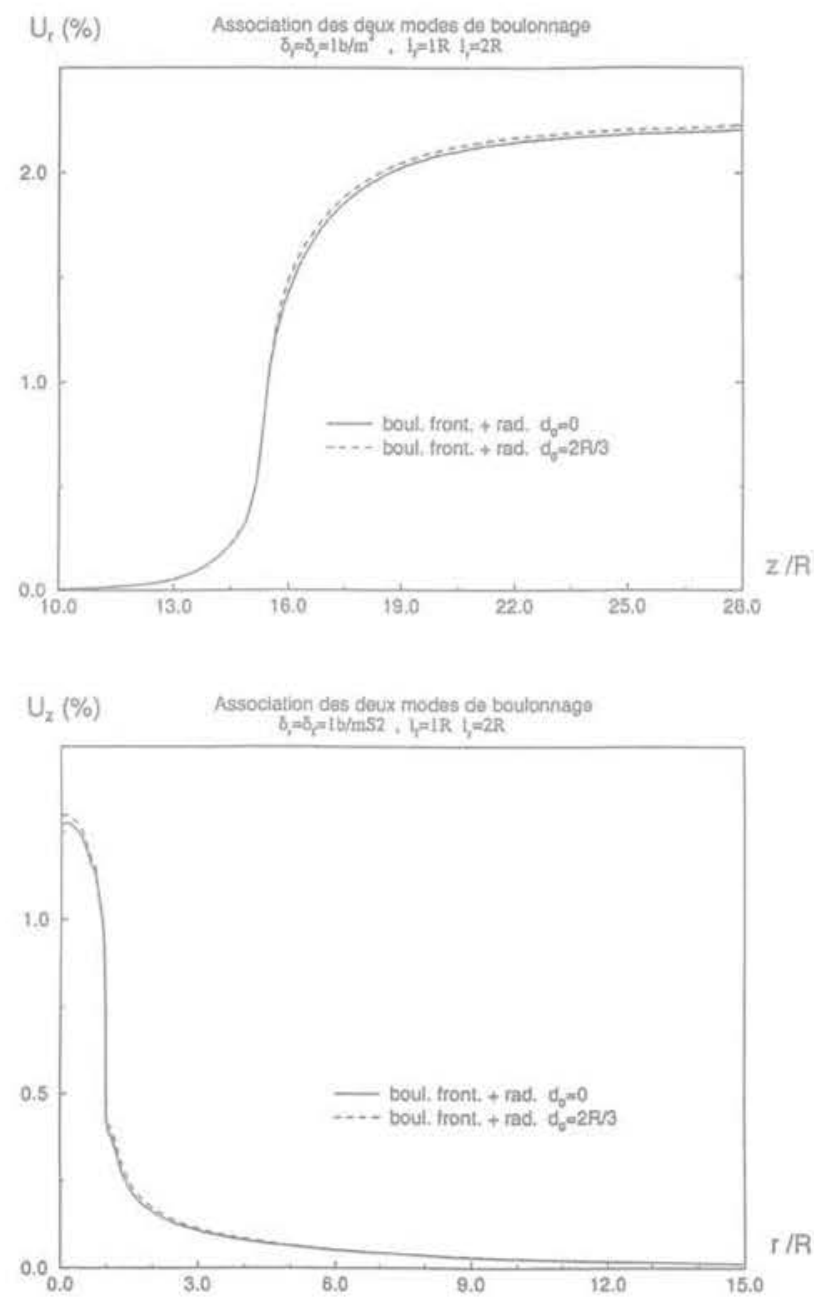

FIG.12. Convergences radiale (a) et axiale (b) pour l'association des deux modes de boulonnage : $d_{0}=2 R / 3$.

Radial (a) and axial (b) convergence when associating radial and facing bolts : $\mathrm{d}_{0}=2 \mathrm{R} / 3$.
Dans ce cas, la réduction de la convergence radiale est évidemment moindre (par rapport au cas $\mathrm{d}_{0}=0$ ) : elle n'est plus que d'environ $8,5 \%$. Il faut noter que la faible influence de $\mathrm{d}_{0}$ dans le cas étudié, est strictement liée à la nature des caractéristiques mécaniques de la roche considérée. Des calculs effectués avec d'autres caractéristiques (voir à ce sujet Bernaud et al., 1995) montrent une influence plus marquée de la distance de pose sur la convergence radiale.

Par contre, si $d_{0} n^{\prime}$ a pratiquement aucune influence sur la convergence axiale, ceci s'explique aisément par le fait que c'est le renforcement frontal (et non radial sur lequel on agit en changeant $\mathrm{d}_{0}$ ) qui est responsable de la réduction de cette convergence.

\section{8}

\section{Prise en compte d'un soutènement en paroi}

En sus des deux modes de boulonnage, nous considérons maintenant le cas où un soutènement élastique est posé sur le pourtour de la paroi du tunnel.

On notera d la distance de pose du soutènement au front (Fig. 13), èt $\mathrm{K}_{\mathrm{s}}$ sa rigidité globale (facteur de pro-

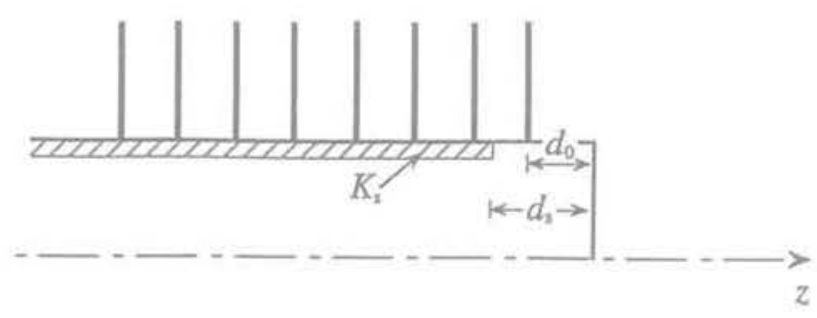

FG.13 Le cas d'un soutènement en paroi. Lining around the tunnel wall.

portionnalité entre la pression exercée sur le soutènement et le déplacement radial, rapporté à $\mathrm{R}$, de celuici). Nous exarninerons les deux cas correspondant à un soutènement de raideur moyenne $\mathrm{K}_{\mathrm{c}}=360 \mathrm{MPa}$, et forte $\mathrm{K}_{\mathrm{g}}=3600 \mathrm{MPa}$.

Le contact soutènement/massif est supposé à adhérence parfaite.

\section{1}

\section{Influence du renforcement frontal sur l'équilibre d'un tunnel soutenu en paroi}

Afin d'appréhender le couplage entre les deux modes de renforcement, nous considérons le cas d'un tunnel pour lequel les boulons frontaux sont associés à un soutènement en paroi. Sauf mention contraire, on prendra $\delta_{\mathrm{f}}=1 \mathrm{~b} / \mathrm{m}^{2}$ et $\mathrm{l}_{\mathrm{f}}=\mathrm{R}$.

Les figures 14 -a et 14 -b montrent les résultats correspondant au cas d'un soutènement de raideur 


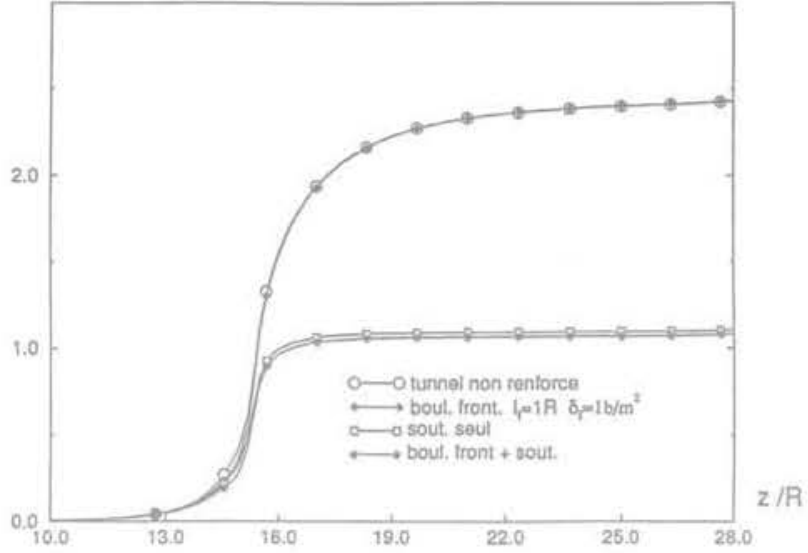

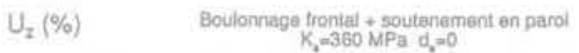

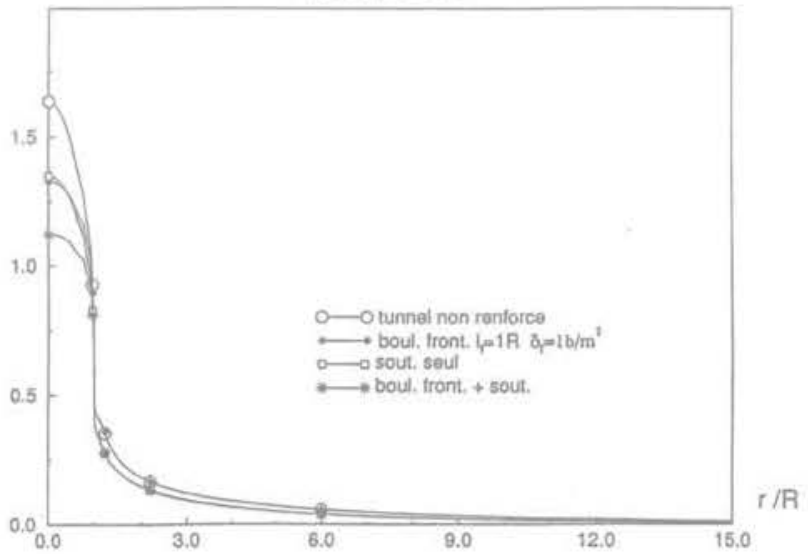

FG.14 Influence du boulonnage frontal sur les convergences du tunnel soutenu. Influence of the facing bolts on the convergence of a tunnel with lining.

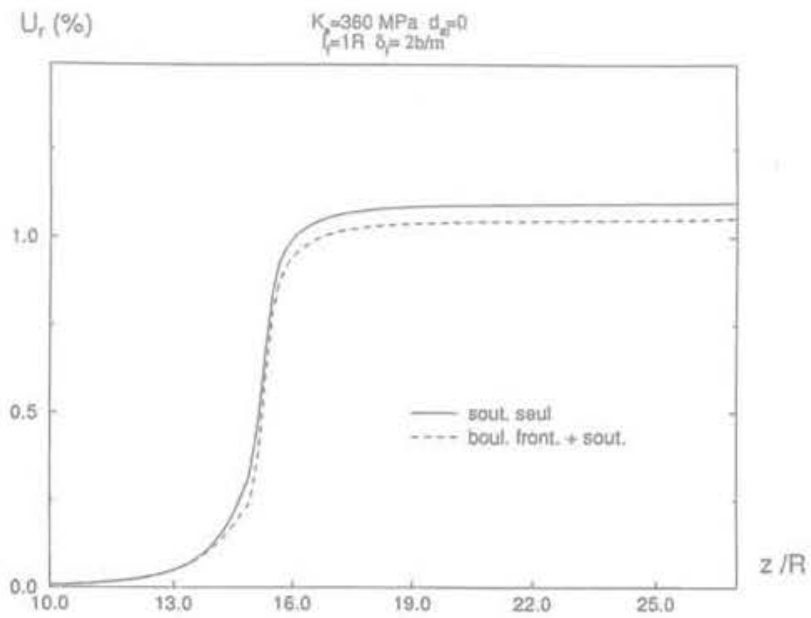

FiG.15 Influence de la densité $\delta$, sur l'interaction soutènement-boulons au front.

Influence of the density $\delta_{f}$ on the interaction between lining and facing bolts.

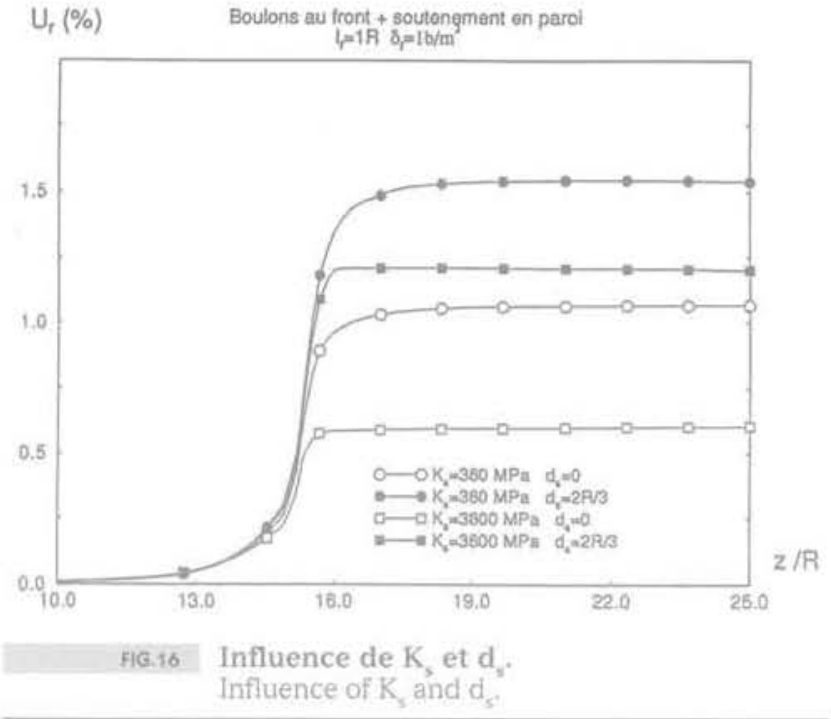

moyenne $\left(\mathrm{K}_{\mathrm{s}}=360 \mathrm{MPa}\right)$ disposé à $\mathrm{d}_{\mathrm{s}}=0$. Ces deux figures semblent indiquer que la présence des boulons au front influe sur les convergences radiale et axiale. En effet, la réduction de celles-ci respectivement loin du front et en $r=0$ sont de :

- $54 \%$ et $18 \%$ pour le soutènement en paroi seul;

$-57 \%$ et $31 \%$ pour l'association soutènement-boulons au front.

Ce résultat suggère que lors de l'application d'une méthode de type convergence-confinement $\left(\mathrm{P}_{1}=f\left(\mathrm{U}_{i}\right)\right)$, la valeur de $u_{0}$ (convergence acquise au moment de la pose du soutenement) dépend aussi des caractéristiques du boulonnage au front.

L'écart au niveau de la convergence radiale (quoique faible) peut s'expliquer de la manière suivante : on a vu auparavant que la présence des boulons frontaux seuls n'affectait celle-ci qu'en la réduisant au voisinage du front. L'ouvrage, lors de la pose du soutènement, "se souvient 》 de cette réduction en la « reportant $\cdots$ au fur et à mesure que le front avance et on la récupère en fin de creusement.

Cette influence au niveau de la convergence radiale est d'autant plus importante que la densité du boulonnage frontale est grande. A titre d'exemple, nous représentons sur la figure 15 les résultats obtenus avec une densité $\delta_{\mathrm{f}}=2 \mathrm{~b} / \mathrm{m}^{2}$.

L'influence de la distance de pose, ainsi que de la rigidité du soutènement, est illustrée sur la figure 16 où 1 , est pris égal à $R$.

\section{2}

\section{Association des boulonnages radial/frontal et d'un soutènement en paroi}

Pour clore l'étude paramétrique entreprise, nous présentons les résultats d'un calcul complet où tous les modes de renforcement sont associés : boulonnage radial et frontal, et soutènement en paroi. Ce dernier, 

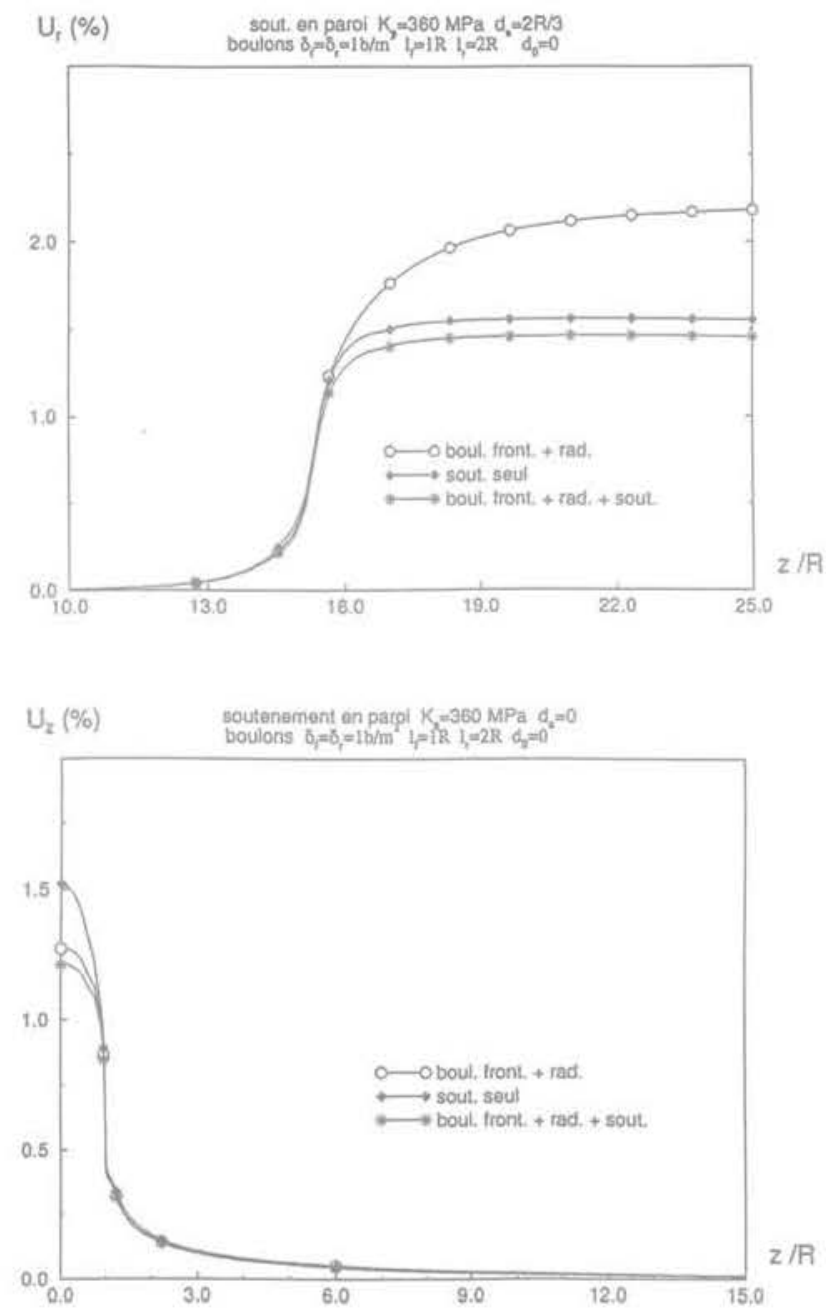

FG.17 Association des deux modes de boulonnage au soutènement. Radial and facing bolts associated with lining.

de rigidité moyenne $\mathrm{K}_{\mathrm{s}}=360 \mathrm{MPa}$, est placé à $\mathrm{d}=0 \mathrm{du}$ front. On prendra $\delta_{r}=\delta_{f}=1 \mathrm{~b} / \mathrm{m}^{2}, l_{f}=R$ et $I_{f}=2 R$. La distance de pose des boulons radiaux est $\mathrm{d}_{0}=2 \mathrm{R} / 3$.

Au-delà des résultats eux-mềmes (Fig. 17-a et 17-b), cet exemple illustre bien la souplesse, et la capacité de la modélisation et de la méthode numérique associée à " gérer » des comportements de natures différentes (roche, matériau homogénéisé, soutènement), ainsi qu'un phasage assez complexe (avancement du front, $\mathrm{d}_{\mathrm{o}} \neq \mathrm{d}_{\mathrm{s}}$ ).

Pour en revenir aux résultats, les valeurs de la convergence radiale loin du front et de la convergence axiale en $\mathrm{r}=0$ pour les différentes configurations de renforcement sont les suivantes :

(1) $2,2 \%$ et $1,27 \%$ pour le cas boulons radiaux + frontaux

(2) $1,55 \%$ et $1,52 \%$ pour le cas soutènement seul;

(3) $1,45 \%$ et $1,21 \%$ pour l'association boulons/soutènement.

Ces valeurs illustrent bien l'interaction entre les différents renforcements. En effet, le classement des convergences radiales par ordre décroissant correspond à la suite (1), (2), (3), alors que pour les convergences axiales il correspond à la suite (2), (1), (3).

\section{Conclusion}

A partir de l'idée heuristique que le massif boulonné peut être considéré comme macroscopiquement (à l'échelle de l'ouvrage) homogène anisotrope, une approche numérique utilisant la technique d'homogénéisation des milieux périodiques a été développée. L'apport essentiel de la démarche est d'éviter les difficultés de discrétisation, sources de problèmes numériques importants, qu'une approche directe par éléments finis implique. L'élaboration de cette méthode a nécessité l'implantation des relations de comportement du matériau homogénéisé dans un code numérique adapté à la modélisation des phases de creusement d'un tunnel et au calcul de sa convergence au fur et à mesure que le front avance.

Bien que l'analyse ait été limitée aux conditions d'axisymétrie, des indications importantes ont pu être dégagées quant aux paramètres pertinents gouvernant le problème, fournissant des éléments précieux pour le choix d'un schéma de renforcement optimal. En particulier, l'étude présentée a mis en évidence le caractère fort couplé entre le massif, les boulons et le soutènement en paroi. Ces composantes interagissent via les paramètres caractérisant chacune d'elles. On conçoit bien alors, que ce type de problème ne peut être abordé de manière satisfaisante qu'à travers une méthode numérique susceptible de tenir compte de ce caractère, qui passe par une modélisation correcte du phasage de l'ouvrage. Il faut noter qu'à ce stade, l'étude doit être étendue à d'autres jeux de données (plus particulièrement celles relatives à la roche), et la validation de l'approche reste à confirmer par comparaison avec des résultats expérimentaux.

Les développements envisageables à court ou moyen terme sont :

- l'application de la méthode à des situations de géométrie et chargement plus complexes ne satisfaisant pas les conditions d'axisymétrie : champ de contrainte initial anisotrope, section de tunnel non circulaire, dissymétrie du boulonnage radial, etc. Le principe de la démarche demeurant le même, une première étape visant à étendre son champ d'application a déjà été entreprise. Il s'agit de la mise en œuvre en condition de déformation plane, ce qui permet de s'affranchir de certaines hypothèses sur la géométrie et le chargement évoquées ci-dessus;

- la mise en cuuvre de la méthode sur des ouvrages géotechniques dépassant le cadre des tunnels boulonnés, et concernant par exemple le cas général d'ouvrages en sols renforcés. En effet, la méthode décrite dans cet article semble tout à fait adaptée à l'évaluation des champs de contraintes et déplacements de telles structures sous l'action de leur chargement : talus cloués ou en terre armée avec ou sans modélisation des phases d'excavation, fondations sur sol renforcé par une densité suffisante de micropieux, etc. 
Bernaud D., de Buhan P., Maghous S. - Numerical simulation of the convergence of a bolt-supported tunnel through a homogenization method. Int. J. Num. Anal. Meth. Geomech, 19, 1995, 267-288.

Bernaud D. - Tunnels profonds dans les milieux viscoplastiques : approches expérimentale et numérique. Thèse Ecole Nationale des Ponts et Chaussées. Paris, France, 1991.

Bernaud D, Rousset G. - L'essai de soutènement à convergence contrôlée : résultats et modélisation. Proc. Int. Symp. Geotech. Eng. Hard Solls-Soft Rocks Athens, 1993, 2, 1381-1391.

De Buhan P. - Approche fondamentale du calcul à la rupture des ouvrages en sols renforcés, Thèse d'État, Paris VI, 1986

De Buhan P., Mangiavacchi R., Nova R. Pelligrini G., Salençon J. - Yield design of reinforced earth walls by a homogenization method. Geotechnique, 39, 1989, 189-201.

De Buhan P., Salençon J, - Yield strength: of reinforced soils as anisotropic media.
Yielding damage and failure of anisotropic solids, Éd. J.P. Boehler, 1990, 791803.

De Buhan P., Taliercio A. - A homogenization approach to the yield strength of composite materials. European J. of Mech.. A/Solids, 10, 1991 129-154.

Gerrard C.M. - Equivalent elastic moduli of a rockmass consisting of orthorombic layers. Int. J. Rock Mech. Min. Sci. Geomech. Abstr., 19, 1982, 9-14.

Gerrard C.M. - Reinforced soil : an orthorombic material. Int. J. Geotechnical. Eng. Div. ASCE, 108, 1982, 1460-1474.

Greuell E. - Étude du soutènement des tunnels par boulons passifs dans les sols et les roches tendres, par une méthode d'homogénéisation. Thèse École polytechnique, Palaiseau. France.

Greuell E., de Buhan P., Panet M., Salencon J. - Behaviour of tunnels reinforced by untensioned bolts. 13th Int. Conf. on Soil Mech. and Found. Eng., New Delhi, 1994.

Hanafy E.A., Emery J.J. - Advancing face simuation of tunnel excavation and lining placement. In : Proc. 13th Canadian Rock Mechanics : Underground Rock Engineering, Toronto, 1980.

Nguyen Q.S. - On the elastic plastic initialboundary value problem and its numerical integration. Int. J. Num. Meth. Eng. 11, 1977, 817-832.

Panet M., Guellec P. - Contribution à l'étude du soutènement d'un tunnel à l'arrière clu front de taille. Proc. 3rd Int. Cong. Rock Mech., Denver, 1974, 2B.

Sawicki A. - On application of effective moduli theory to layered soil, Hydrot. Trans, 39, 1978, 3-13.

Wullschläger D., Natau O. - Studies of the composite system of rockmass and nonprestressed grouted rockbolts. Proc. Int. Symp. Rock Bolting, Abisko, 1983.

Wullschläger D., Natau O. - The bolted rockmass as an anisotropic continuum. Material behaviour and design suggestion for rock cavities. Proc. 6th Int. Cong. Rock Mech., Montreal, 1987, 1321-1324. 